\title{
ORIGINAL CONTRIBUTION Creating a Diploma Supplement Linked to Degree-Awarding Policies When the
Curriculum Comprises Mostly of Elective Subjects
}

\author{
Yoshioka Tsuyoshi ${ }^{1 *}$, Teruya Kensaku ${ }^{2}$ \\ 1,2 Teikyo Heisei University, Tokyo, Japan
}

\begin{abstract}
This study aims at defining a remodeled adjusted value for Learning Degree value Graph (LDG) and proposing a method to recalculate the Learning Degree (LD). The Diploma Supplement (DS) is an attachment to a degree certificate that enhances transparency by clearly indicating the contents acquired. DS for a curriculum mostly is comprised of electives and cannot fairly represent student abilities. This study uses a DS creation method linked to degree-awarding policies—commonly called "Diploma Policies" (DP) in Japan. Specifically, each subject is associated with a plurality of DP items and a student's contribution rate in a subject (subject learning rate) is set based on the contents of the DP items. The DS is created by multiplying the number of acquired credits by the subject learning rate of each DP and the learning evaluation level as the learning degree of each DP item. If all students are taking the same subjects, then a fair DS can be created by this method by calculating the LD. As a result, a method for creating a DS linked to DPs that accurately represents student abilities even when the curriculum is mostly composed of electives is successfully developed. This method could be helpful as the DS here reflects a fair evaluation of student's degree regardless of the subjects studied.
\end{abstract}

Index Terms - DS, DP, DS linked to DPs, Elective Subjects.

Received: 13 August 2020; Accepted: 21 November 2020; Published: 19 December 2020

\section{Introduction}

In 1999, the DS (European Commission, n.d.) was introduced to European universities as part of the Bologna Process (Wachter, 2004). The Bologna Process is an agreement between European countries aimed at ensuring that higher education degrees awarded in any of the signatory countries are of the same standard and comparable level in all countries. Meanwhile, the DS is an attachment to a degree certificate that enhances transparency by clearly indicating the contents acquired. It is written in English and follows a standard template to describe the degree, qualifications, and awarding agency (Cyprus, 2013). The DS has been introduced in many European countries (Heryandi \& Afrianto, 2019) and have been extensively studied because of its increasing importance around the world (Australian Government Department of Education, Employment and Workplace Relations, 2010; Education, Audiovisual and Culture Executive Agency, 2017a,b,c,d).

In Japan, DSs have been introduced or considered for introduction at many universities in the past few years (Advanced Institute of Industrial Technology, 2016; Yumoto \& Sumida, 2019; Fukahori, 2019; Tokyo City University, n.d.; Tokyo University of Foreign Studies, n.d.). Although each university may prepare a DS by its own methods, this study uses a DS creation method linked to degree-awarding policies that are commonly called DPs in Japan. Specifically, each subject is associated with a plurality of DP items, and a student's contribution rate in a subject (subject learning rate) is set based on the contents of the DP items. The DS is created by multiplying the number of acquired credits by the subject learning rate of each DP and the learning evaluation level (e.g., grade point average) as the LD of each DP item.

If all students are taking the same subjects, then a fair DS can be created by this method by calculating the LD. However, if a student's curriculum is mainly composed of elective subjects, then the subjects taken vary by student. A fair DS would only take into account the required subjects; therefore, a DS in the case where a curriculum is mostly comprised of electives does not fairly represent student abilities.

In this study, we developed a method for creating a DS linked to DPs that can accurately represent student abilities even when their curriculum is mostly composed of elective subjects. Using this method, it is possible to create a DS that reflects a fair evaluation of a student's degree regardless of the subjects they have taken, even if they have only completed the minimum number of credits required for graduation.

\section{Methodology}

In this section, we show how to create a DS linked to DP items that accurately represent student abilities in a curriculum. First, a DS creation method is described in which the curriculum comprises only required subjects that link to DP items. Next, we propose a new DS creation method in the curriculum composed mostly of elective subjects.

\footnotetext{
Email: t.yoshioka@thu.ac.jp
} 


\section{DS for required subjects}

In this section, the DS creation method links a curriculum comprised only of required subjects to DP items. This method is widely used in Japan (Advanced Institute of Industrial Technology, 2016; Yumoto \& Sumida, 2019; Tokyo City University, n.d.; Tokyo University of Foreign Studies, n.d.), but summarize it. This is because of the necessary discussion in the next section for developing the DS creation method when the majority of subjects are electives. It is assumed that the DPs comprises six items, as shown in Table I. It is also assumed that 124 total credits are required for graduation (this is the threshold that Japanese universities generally set), there are a total of 60 subjects, and the curriculum requires students to complete courses in each subject, as shown in Table II.

Table I

Items of DPs

\begin{tabular}{ll}
\hline DP No. & Item \\
\hline DP 1 & Major \\
DP 2 & Liberal Arts \\
DP 3 & Information Literacy \\
DP 4 & Speaking \\
DP 5 & Writing \\
DP 6 & Reading \\
\hline
\end{tabular}

Next, column (a) of Table III details the ratios-or "subject learning rates" (SL rate)-at which each subject contributes to each DP item. The sum of these ratios is $100 \%$. In column (b) of Table III, learning evaluation points (LE level, e.g., grade point average) are shown. LE level is widely used in Japanese universities. The LE scale is divided into 5 levels from 0 to 4 , with the highest value indicating a good evaluation Table III. Further, LD value obtained by multiplying the number of credits, SL rate, and LE level of each subject is shown in column (c) of Table III. The bottom row of this table shows the result when the LE level of all subjects is the maximum value.

Table II

Subject Name and Number of Credits

\begin{tabular}{ll}
\hline Subject name & Number of credits \\
\hline Sub-01 & 1 \\
Sub-02 & 1 \\
Sub-03 & 2 \\
Sub-04 & 4 \\
$\cdot$ & $\cdot$ \\
Sub-60 & $\cdot$ \\
Total number of credits & 2 \\
\hline Note: All subjects are required subjects. & 124 \\
\hline
\end{tabular}

Meanwhile, in Fig. 1, the total value of each DP item calculated above is shown in a radar chart. In this case, even if the LE level of all subjects is a maximum of 4 points, the shape of the radar chart is distorted because the total LD values of each DP item is different. Therefore, in the radar chart, the total value of each LD value is multiplied by the value in Table IV so that the final maximum LD value of all DP items becomes 100 . We name this the adjusted value for the LD value graph (adjusted value for LDG). If the LE level of each subject is not the maximum value, then the shape of the radar chart changes according to the obtained value.

Table III

Calculation example of LD

\begin{tabular}{|c|c|c|c|c|c|c|c|c|c|c|c|c|c|c|}
\hline \multirow[t]{2}{*}{ Subject name } & \multirow[t]{2}{*}{ Number of credits } & \multicolumn{6}{|c|}{ (a) SL rate of each DP\% } & \multirow{2}{*}{$\begin{array}{l}\text { (b) } \\
\text { LE level }\end{array}$} & \multicolumn{6}{|c|}{ (c) LD value of each DP item } \\
\hline & & DP 1 & DP 2 & DP 3 & DP 4 & DP 5 & DP 6 & & DP 1 & DP 2 & DP 3 & DP 4 & DP 5 & DP 6 \\
\hline Sub-01 & 1 & 10 & 30 & 0 & 20 & 20 & 20 & 4 & 0.4 & 1.2 & 0.0 & 0.8 & 0.8 & 0.8 \\
\hline Sub-02 & 1 & 0 & 20 & 50 & 10 & 10 & 10 & 4 & 0.0 & 0.8 & 2.0 & 0.4 & 0.4 & 0.4 \\
\hline Sub-03 & 2 & 10 & 50 & 10 & 10 & 10 & 10 & 4 & 0.8 & 4.0 & 0.8 & 0.8 & 0.8 & 0.8 \\
\hline Sub-04 & 4 & 80 & 10 & 10 & 0 & 0 & 0 & 4 & 12.8 & 1.6 & 1.6 & 0.0 & 0.0 & 0.0 \\
\hline . & . & . & . & . & . & . & . & . & . & . & . & . & . & . \\
\hline . & . & . & . & $\cdot$ & . & . & . & . & . & . & . & . & . & . \\
\hline Sub-60 & 2 & 80 & 10 & 10 & 0 & 0 & 0 & 4 & 12.8 & 1.6 & 1.6 & 0.0 & 0.0 & 0.0 \\
\hline Total & 124 & - & - & - & - & - & - & - & 133.2 & 135.6 & 84.4 & 47.6 & 47.6 & 47.6 \\
\hline
\end{tabular}

Note: All subjects are required subjects.

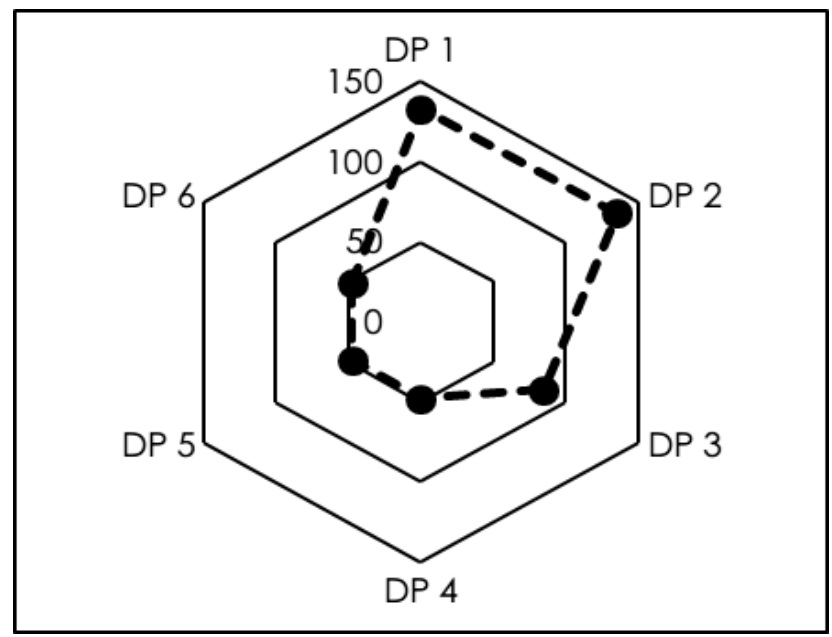

Fig. 1. LD value graph 
Fig. 2 is created by multiplying the value of each DP item in Fig. 1 by the corresponding adjusted value for LDG (Table IV). That is, the adjusted value for LDG is represented by the equation 1 . Not surprisingly, if the LE level of all subjects reaches the maximum value of 4 points, then the LD of each DP item is 100 .

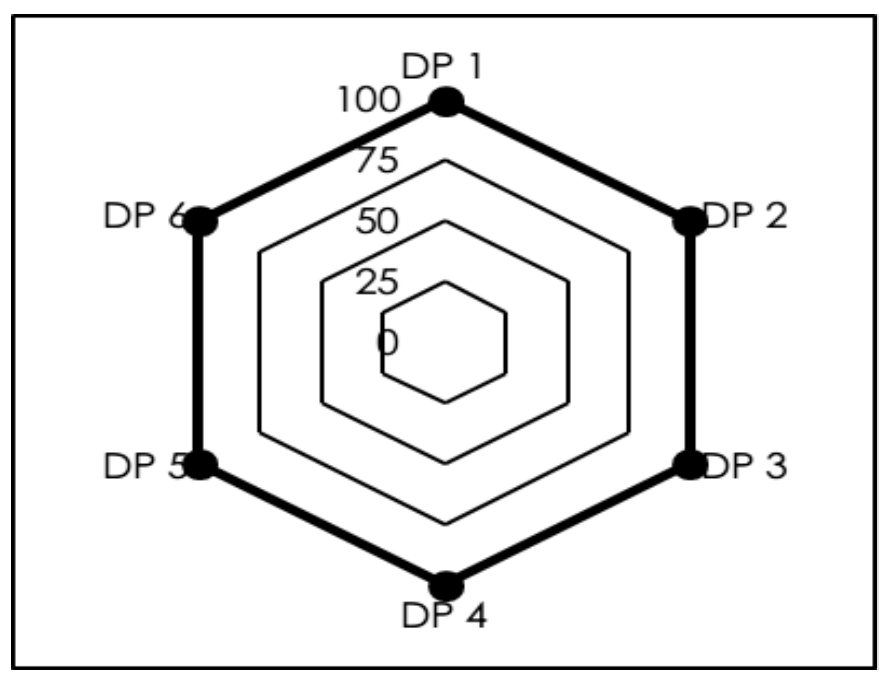

Fig. 2. Adjusted $L D$ value graph

Table IV

The adjusted value for LDG

\begin{tabular}{ll}
\hline DP & The adjusted value for LDG \\
\hline DP 1 & $100 / 133.2$ \\
DP 2 & $100 / 135.6$ \\
DP 3 & $100 / 84.4$ \\
DP 4 & $100 / 47.6$ \\
DP 5 & $100 / 47.6$ \\
DP 6 & $100 / 47.6$ \\
\hline Note: All subjects are required subjects.
\end{tabular}

The equation of adjusted value for LDG is:

$$
\text { Adjusted value for } L D G=\frac{100}{(\text { Total LD value of each DP })}
$$

So far, we have introduced a method for creating a DS that links a curriculum in which all subjects are required DP items. However, in a curriculum mainly composed of elective subjects, it becomes more difficult to fairly express a student's abilities using this method. In the next section, we elaborate on how to create a DS for a curriculum dominated by elective subjects.

\section{Creating a New DS by Linking Mostly Elective Subjects}

In this section, the DS creation method links a curriculum where the majority of subjects are electives. As in the previous section, it is assumed that DPs consists of six items. In this case, it is assumed that most of the subjects set in the curriculum are elective subjects and that there are 100 subjects. In addition, as Table $V$ shows, it is assumed that the total number of credits is 200 when all the subjects are taken. As stated above, the number of credits required for graduation is 124; thus students do not need to take courses in every subject. Students can also graduate with more than 124 credits. Therefore, the number of subjects students take as well as the types of subjects they take can differ; accordingly, the earlier DS method cannot be reasonably applied to this case. Hence, we modified the creation method described in previous section.

Table V

Subject name and number of credits

\begin{tabular}{ll}
\hline Subject name & Number of credits \\
\hline Sub-01 & 1 \\
Sub-02 & 1 \\
Sub-03 & 2 \\
Sub-04 & 4 \\
$\cdot$ & $\cdot$ \\
$\cdot$ & $\cdot$ \\
. & $\cdot$ \\
Sub-100 & 2 \\
Total number of credits & 200 \\
\hline Note: All subjects are elective subjects.
\end{tabular}

Table VI shows a calculation example of the LD value similar to Table III. In this case, however, a weighting coefficient was added to the column (d) of Table VI. The reason for the addition was that, even if the number of credits assigned to subjects remains the same, it is necessary to consider that some subjects may be more important or less important when arriving at a DS based on DP items. Although the weighting coefficient is basically set to 1 , an arbitrary value is set according to the difference in importance. In this case, the LD value is calculated by a value obtained by multiplying the number of credits, weighting coefficient, SL rate, and LE level of each subject. The sum of the LD values of each DP item in all subjects is shown in the bottom row of column (c) of Table VI.

In this case, the adjusted value for LDG can be calculated in the same manner as it was for Table IV. It is also possible to create a radar chart from the values. However, in this calculation method, when 100 subjects for 200 credits are taken and the LE level of all subjects is the maximum, the sum of the LD values of the DP items becomes $100 \%$. In other words, even if the student earns the 124 credits needed to graduate and achieved the highest grades in every subject, the DS result would be $62 \%$ (=124/200). 
Table VI

Calculation example of LD

\begin{tabular}{|c|c|c|c|c|c|c|c|c|c|c|c|c|c|c|c|}
\hline \multirow[t]{2}{*}{ Subject name } & \multirow{2}{*}{$\begin{array}{l}\text { Number of } \\
\text { credits }\end{array}$} & \multirow{2}{*}{$\begin{array}{l}\text { (d) } \\
\text { Weight }\end{array}$} & \multicolumn{6}{|c|}{ (a) SL rate of each DP\% } & \multirow{2}{*}{$\begin{array}{l}\text { (b) } \\
\text { LE level }\end{array}$} & \multicolumn{6}{|c|}{ (c) LD value of each DP item } \\
\hline & & & DP 1 & DP 2 & DP 3 & DP 4 & DP 5 & DP 6 & & DP 1 & DP 2 & DP 3 & DP 4 & DP 5 & DP 6 \\
\hline Sub-01 & 1 & 1 & 10 & 30 & 0 & 20 & 20 & 20 & 4 & 0.4 & 1.2 & 0.0 & 0.8 & 0.8 & 0.8 \\
\hline Sub-02 & 1 & 2 & 0 & 20 & 50 & 10 & 10 & 10 & 4 & 0.0 & 0.8 & 2.0 & 0.4 & 0.4 & 0.4 \\
\hline Sub-03 & 2 & 1 & 10 & 50 & 10 & 10 & 10 & 10 & 4 & 0.8 & 4.0 & 0.8 & 0.8 & 0.8 & 0.8 \\
\hline Sub-04 & 4 & 0.5 & 80 & 10 & 10 & 0 & 0 & 0 & 4 & 12.8 & 1.6 & 1.6 & 0.0 & 0.0 & 0.0 \\
\hline . & . & . & . & . & . & . & . & . & . & . & . & . & . & . & . \\
\hline . & . & . & . & . & . & . & . & . & . & . & . & . & . & . & . \\
\hline$\cdot$ & . & . & . & . & . & . & . & . & . & . & . & . & . & . & . \\
\hline Sub-60 & 2 & 1 & 80 & 10 & 10 & 0 & 0 & 0 & 4 & 12.8 & 1.6 & 1.6 & 0.0 & 0.0 & 0.0 \\
\hline Total & 200 & - & - & - & - & - & - & - & - & 202.8 & 223.2 & 147.2 & 82.0 & 76.4 & 80.4 \\
\hline
\end{tabular}

Note: All subjects are elective subjects. Students can graduate if they earn 124 or more credits.

Thus, it is essential to adjust the LD value of each DP item to $100 \%$, if the student acquires the 124 credits required for graduation with the highest LE level in a curriculum consisting of 200 credits. Hence, Table VII shows an improved version of the adjusted value for the LDG in Table IV. This is denominated the Remodeled adjusted value for LDG. It is shown in the equation 2.
Table VII

Remodeled adjusted value for LDG

\begin{tabular}{ll}
\hline DP & Remodeled adjusted value for LDG \\
\hline DP 1 & $100 / 82.0^{*} 200 / 124$ \\
DP 2 & $100 / 76.4^{*} 200 / 124$ \\
DP 3 & $100 / 80.4^{*} 200 / 124$ \\
DP 4 & $100 / 147.2 * 200 / 124$ \\
DP 5 & $100 / 223.2 * 200 / 124$ \\
DP 6 & $100 / 202.8 * 200 / 124$ \\
\hline
\end{tabular}

Remodeled adjusted value for $L D G=\frac{100}{(\text { Total LD value of each DP })} \times \frac{(\text { Total number of credits })}{(\text { Credits required for graduation })}$

\section{Discussion}

The Remodeled adjusted value for LDG is the adjusted value for LDG multiplied by the Total number of credit/Credits required for graduation. By using this remodeled adjusted value for LDG, it is possible to create a fair DS linked to DPs even in a curriculum in which most subjects are elective subjects. However, when using this method, it is important to note that if a student completes more than the required number of credits for graduation, then the value displayed on the radar chart may exceed $100 \%$. In other words, if the LE level of all 124 credits required for graduation was the highest, it would be exactly $100 \%$, but if it exceeded 124 credits, then it would exceed $100 \%$. The method of setting the remodeled adjusted value for LDG can be used not only in a curriculum comprising a majority of elective subjects but also in a curriculum in which all subjects are required. Therefore, regardless of the curriculum, using a remodeled adjusted value for LDG can create a fair DS.

\section{Limitations and Future Research Directions}

This study proposed a new methodology to create DS which fairly represents the abilities of students. However, this method has not been tested and verified widely. In future, scholars are encourgaed to test the current method in different institutions and enhance its credibility.

\section{Conclusion}

This paper presents a method for creating a DS linked to DPs that accurately represents student ability, even in a curriculum mostly comprised of elective subjects. If the gremodeled adjusted value for LDG is used, then a fair DS can be created regardless of whether the curriculum is comprised of many elective subjects or whether the student earns more than the number of credits required for graduation. Using this method, it is possible to create a DS that reflects a fair evaluation of a student's degree regardless of the subjects they have taken, even if they have only completed the minimum number of credits required for graduation.

\section{References}

Advanced Institute of Industrial Technology. (2016). Mechanism for squeezing (diploma supplements, etc.). Retrieved from https://bit $.1 \mathrm{y} / 330 \mathrm{KuLx}$

Australian Government Department of Education, Employment and Workplace Relations. (2010). Higher education diploma supplements among APEC member economies (Tech. Rep.). Melbourne, Australia: AsiaPacific Economic Cooperation Secretariat.

Cyprus, A. C. (2013). Diploma supplement. Retrieved from https:// bit.1y/2ITrkjs

Education, Audiovisual and Culture Executive Agency. (2017a). Overview of higher education system in Egypt (Tech. Rep.). Brussels, Germany: EACEA.

Education, Audiovisual and Culture Executive Agency. (2017b). Overview of higher education system in Lebanon (Tech. Rep.). Brussels, Germany: EACEA

Education, Audiovisual and Culture Executive Agency. (2017c). Overview of higher education system in Palestine (Tech. Rep.). Brussels, Germany: EACEA.

Education, Audiovisual and Culture Executive Agency. (2017d). Overview of higher education system in Tunisia (Tech. Rep.). Brussels, Germany: EACEA.

European Commission. (n.d.). Diploma suplements. Retrieved from https://bit.1y/3nCVJBR

Fukahori, S. (2019). The Japanese higher education quality assurance system's challenge revealed through the Japanese diploma supplement. Journal of Japanese Society for Engineering Education, 67, 22-29. doi:https://doi.org/10.4307/jsee.67.1_22 
Journal of Management Practices, Humanities and Social Sciences 4(2) 45-49

Heryandi, A., \& Afrianto, I. (2019). Online diploma supplement information system modelling for indonesian higher education institution. IOP Conference Series: Materials Science and Engineering, 662(2), 22-29.

Tokyo City University. (n.d.). Acceleration program for university education rebuilding business overview. Retrieved from https://bit.ly/ 35Lqajf

Tokyo University of Foreign Studies. (n.d.). TUFS diploma supplement. Retrieved from https://bit.1y/332YYL8
Wachter, B. (2004). The Bologna process: Developments and prospects. European Journal of Education, 39, 265-273.

Yumoto, M., \& Sumida, A. (2019). Design of diploma supplement for finding students themselves and for improving their academic outcomes. Journal of Japanese Society for Engineering Education, 67(1), 28-33. doi:https://doi.org/10.4307/jsee.67.1_28 